\title{
Risk factors for thromboembolism in burn patients admitted to the burn unit at King Abdulaziz Medical City, Riyadh, Kingdom of Saudi Arabia
}

Thamer A. Althunayan, MBBS, Saad M. AlQarni, MBBS, Waleed A. Mohsenh, MBBS, Ahmed M. Alkhalifah, MBBS, Abdullmajeed N. Alsadi, MBBS, Omar S. Alrushid, MBBS, Mohammad M. Al-Qattan, MBBS.

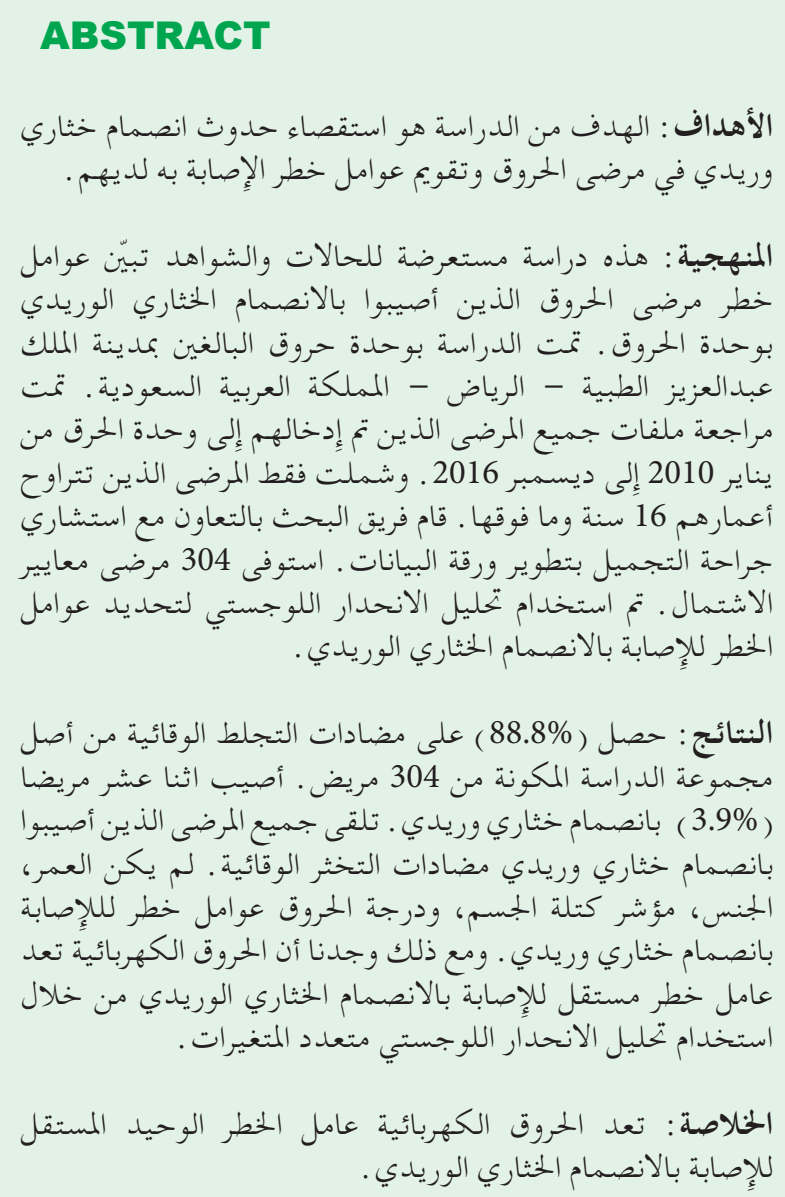

Objectives: To investigate the prevalence and associated risk factors for venous thromboembolism (VTE) in burn patients.

Methods: This is a cross-sectional study eliciting the risk factors in burn patients who developed thromboembolism. The study took place at the Adult

Burn Unit, King Abdulaziz Medical City (KAMC), Riyadh, Saudi Arabia. Charts of all patients admitted to the burn unit from January 2010 to December 2016 were reviewed. Only patients 16 years of age and older were included. The research team with a consultant plastic surgeon developed the data sheet. A total of 304 patient records met the inclusion criteria. Logistic regression analysis was used to determine the risk factors for developing VTE.

Results: Out of the entire study group of 304 patients, the majority $(88.8 \%)$ of the participants received prophylactic anticoagulation. Twelve patients (3.9\%) developed VTE. All patients who developed VTE received prophylactic anticoagulation. Age, gender, body mass index, and degree of burn were not risk factors for VTE. However, electric burns were found to be an independent significant risk factor for developing VTE using a multivariate logistic regression.

Conclusion: Electric burns were found to be the only independent significant risk factor of developing VTE.

Saudi Med J 2019; Vol. 40 (10): 1027-1031 doi: 10.15537/smj.2019.10.23955

From the College of Medicine (Althunayan, AlQarni, Mohsenh, Alkhalifah, Alsadi, Alrushid), King Saud bin Abdulaziz University for Health Sciences; from the Department of Surgery (Qattan), Ministry of the National Guard Health Affairs, Riyadh, Kingdom of Saudi Arabia, and from the Department of Surgery (Qattan), King Saud University, Riyadh, Saudi Arabia; from the Department of Research (Althunayan, AlQarni, Mohsenh, Alkhalifah, Alsadi, Alrushid, Qattan) King Abdullah International Medical Research Center, Riyadh, Kingdom of Saudi Arabia.

Received 5th March 2019. Accepted 1st September 2019.

Address correspondence and reprint request to: Dr. Thamer A. Althunayan, College of Medicine, King Saud bin Abdulaziz University for Health Sciences, Riyadh, Kingdom of Saudi Arabia.E-mail: thamer.a.t@gmail.com

ORCID ID: https://orcid.org/0000-0002-8681-2346 
T here is an increased risk of venous thromboembolism (VTE) development in burn patients, which is generally attributed to their hypercoagulable state. ${ }^{1}$ Venous thromboembolism, also known as deep vein thrombosis (DVT), mostly occurs in the deep veins of the thighs or legs. The main concern related to the development of DVT is clot fragmentation and embolus formation leading to pulmonary embolism (PE). Burn patients fulfill all 3 factors stated in Virchow's triad of thrombosis which consists of changes in blood flow, injury to the endothelial layer of the vessels, and alterations in the components of blood known as hypercoagulability. ${ }^{2}$ In addition, burn platelet count. ${ }^{1}$ However, the reported rates of VTE among burn patients in the literature varied greatly between $0.25-25 \% .^{3-5}$ Prophylactic anticoagulation for burn patients is still controversial not only because the reported rates are variable, but also because of the fear of complications associated with anticoagulation. ${ }^{1,6-10}$

The aim of this study was to estimate the rate of VTE in burn patients and determine the risk factors associated with such events.

Methods. A cross-sectional study was conducted to assess the rate and risk factors of burn patients who developed thromboembolism. Ethical approval was obtained from King Abdullah International Medical Research Center. Consent from patients was not required. After obtaining the Institutional Review Board (IRB) approval, the Plastic Surgery Division provided a list of patients' medical record numbers admitted to the burn unit from January 2010 to December 2016. The study was conducted in the Adult Burn Center of King Abdulaziz Medical City, Riyadh, Saudi Arabia. The center contains 8 acute intensive care unit (ICU) beds, an operation room, and 3 outpatient clinics.

The study included the medical records of all adult burn patients, 16 years and older, who were admitted to patients, 204 were ineligible due to their age, while 31 patents' files were missing and their status of eligibility is unknown. Hence, the remaining 304 patients made our study population.

This was a chart review and the research team collected the data from patients' files after developing a data sheet. Data collected were the use of prophylactic

Disclosure. Authors have no conflict of interests, and the work was not supported or funded by any drug company. patients frequently have a significant increase in their the burn unit between 2010 and 2016. Of the total 539

anticoagulation, patients' body mass index (BMI), co-morbidities, type of burn, degree of burn, total body surface area of burn (TBSA), development of septicemia, blood transfusions, intubation, and surgical procedures. The outcome variable was developing thromboembolism (DVT or PE).

Statistical analysis. Microsoft Excel was used for data entry, and the data were analyzed by the Statistical Package for Social Sciences for Windows, version 22.0 (IBM Corp, Armonk, NY, USA). Frequencies and percentages were used for categorical data, for example co-morbidities, and gender. Mean and standard deviation were used for numerical data, such as age. For inferential statistics, we used t-test to compare numerical data between patients with and without thromboembolism, and the Fisher-exact test to compare categorical data between patients with and without thromboembolism. We used univariate logistic regression analysis to assess the risk factors, and multivariate logistic regression to assess the independent risk factors. The level of significance in this study was $<0.05$.

Results. The results of the demographic data are shown in Table 1 . Of the overall number of patients (304), 3.9\% (12 patients) developed VTE. All

Table 1 - The results of the demographic patients' data $(\mathrm{N}=304)$.

\begin{tabular}{lrr}
\hline Variables & $\mathbf{n}(\%)$ \\
\hline Gender & 213 & $(70.1)$ \\
Male & 91 & $(29.9)$ \\
Female & 35.13 & $(15.05)$ \\
Age (mean \pm SD) & 112 & $(36.84)$ \\
Obesity & & \\
Type of burn & 210 & $(69.1)$ \\
Flame burn & 43 & $(14.1)$ \\
Scald burn & 30 & $(9.9)$ \\
Chemical burn & 16 & $(5.3)$ \\
Electrical burn & 5 & $(1.6)$ \\
Contact burn & & \\
Degree of burn & 150 & $(49.3)$ \\
2nd degree & 141 & $(46.4)$ \\
3rd degree & 7 & $(2.3)$ \\
4th degree & 228 & $(75.0)$ \\
Lower limb involvement & 125 & $(41.1)$ \\
Intubation during the course of & & \\
treatment & 103 & $(33.9)$ \\
Procedure & 270 & $(88.8)$ \\
DVT prophylaxis & & \\
Drug of DVT prophylaxis & 263 & $(86.5)$ \\
Heparin & 7 & $(2.3)$ \\
Enoxaparin & & \\
\hline Data are presented as numbers and percentage & $(\%)$. \\
$\quad$ DVT - deep vein thrombosis & &
\end{tabular}


patients who developed VTE received prophylactic anticoagulation, heparin was used in 11 patients, and the remaining patient received enoxaparin. Deep vein thrombosis prophylaxis was given to $88.8 \%$ of total

Table 2 - Univariate logistic regression analysis for risk factors of venous thromboembolism among burn patients admitted to the burn unit.

\begin{tabular}{|c|c|c|c|c|c|}
\hline Risk factors & $\begin{array}{r}\text { VTE } \\
\text { n (\%) } \\
\end{array}$ & $\begin{array}{c}\text { No VTE } \\
\text { n (\%) }\end{array}$ & OR & $\begin{array}{c}95 \% \text { CI for } \\
\text { OR }\end{array}$ & $P=$ value \\
\hline $\begin{array}{l}\text { Not taking } \\
\text { thromboembolism } \\
\text { prophylactic } \\
\text { medication }\end{array}$ & 0 & $244(100)$ & 0 & - & 0.998 \\
\hline \multicolumn{6}{|l|}{ Hypertension } \\
\hline Yes & $3(25.0)$ & $38(13.0)$ & 2.23 & $0.58-8.60$ & \multirow{2}{*}{0.245} \\
\hline $\mathrm{No}^{\dagger}$ & $9(75.0)$ & $254(87.0)$ & 1 & & \\
\hline \multicolumn{6}{|l|}{ Diabetes mellitus } \\
\hline Yes & $4(33.3)$ & $40(13.7)$ & 3.15 & $0.91-10.95$ & \multirow{2}{*}{0.071} \\
\hline $\mathrm{No}^{\dagger}$ & $8(66.7)$ & $252(86.3)$ & 1 & & \\
\hline \multicolumn{6}{|c|}{ Ischemic heart disease } \\
\hline Yes & 1 & 14 & 1.81 & $0.22-14.98$ & \multirow{2}{*}{0.584} \\
\hline $\mathrm{No}^{\dagger}$ & $11(91.7)$ & $278(95.2)$ & 1 & & \\
\hline \multicolumn{6}{|l|}{ Obesity } \\
\hline Yes & $8(66.7)$ & $104(37.0)$ & 3.4 & $1.00-11.58$ & \multirow{2}{*}{$0.049^{*}$} \\
\hline $\mathrm{No}^{\dagger}$ & $4(33.3)$ & $177(63.0)$ & 1 & & \\
\hline \multicolumn{6}{|l|}{ Smoking } \\
\hline Yes & 0 & $7(2.4)$ & 0 & - & \multirow{2}{*}{0.999} \\
\hline $\mathrm{No}^{\dagger}$ & $12(100)$ & 285 (97.6) & 1 & & \\
\hline \multicolumn{6}{|l|}{ Gender } \\
\hline Female & $5(41.7)$ & $86(29.5)$ & 1.71 & $0.53-5.54$ & \multirow{2}{*}{0.370} \\
\hline $\mathrm{Male}^{\dagger}$ & $7(58.3)$ & $206(70.5)$ & 1 & & \\
\hline \multicolumn{6}{|l|}{ Patient intubated } \\
\hline Yes & $9(75.0)$ & $116(39.7)$ & 4.55 & $1.21-17.16$ & \multirow{2}{*}{$0.032^{*}$} \\
\hline $\mathrm{No}^{\dagger}$ & $3(25.0)$ & $176(60.3)$ & 1 & & \\
\hline \multicolumn{6}{|l|}{ Septicemia } \\
\hline Yes & $9(75.0)$ & $61(21.2)$ & 11.16 & $2.93-42.50$ & \multirow{2}{*}{$<0.001^{*}$} \\
\hline $\mathrm{No}^{\dagger}$ & $3(25.0)$ & $227(78.8)$ & 1 & & \\
\hline \multicolumn{6}{|l|}{ Degree of burn } \\
\hline 2nd degree ${ }^{\dagger}$ & $3(25.0)$ & $139(50.0)$ & 1 & & \\
\hline 3rd degree & $7(58.3)$ & $134(48.2)$ & 2.42 & $0.61-9.56$ & 0.217 \\
\hline 4th degree & $2(16.7)$ & $5(1.8)$ & 18.53 & $2.51-136.83$ & $0.004^{*}$ \\
\hline \multicolumn{6}{|l|}{ Type of burn } \\
\hline Flame burn ${ }^{\dagger}$ & $7(58.3)$ & $203(83.2)$ & 1 & & \\
\hline Chemical burn & $1(8.3)$ & $29(11.9)$ & 1 & $0.12-8.24$ & 1.000 \\
\hline Electrical burn & $4(33.3)$ & 12 (4.9) & 9.67 & $2.48-37.64$ & $0.001^{*}$ \\
\hline $\mathrm{TBSA} \leq 25^{\dagger}$ & $2(16.7)$ & $165(57.1)$ & 1 & & \\
\hline TBSA $>25$ & $10(83.3)$ & $124(42.9)$ & 6.65 & $1.43-30.91$ & $0.016^{*}$ \\
\hline \multicolumn{6}{|l|}{ Burn \% of leg } \\
\hline$\leq 20^{\dagger}$ & $1(25.0)$ & $72(36.9)$ & 1 & & \\
\hline$>20$ & $3(75.0)$ & $123(63.1)$ & 1.76 & $0.18-17.20$ & 0.629 \\
\hline \multicolumn{6}{|l|}{ Packed $R B C$} \\
\hline$\leq 5^{\dagger}$ & $4(40.0)$ & $54(38.3)$ & 1 & & \\
\hline$>5$ & $6(60.0)$ & $87(61.7)$ & 1.07 & $0.29-3.98$ & 0.915 \\
\hline \multicolumn{6}{|l|}{ Grafting } \\
\hline Yes & $10(83.3)$ & $223(76.6)$ & 1.53 & $0.33-7.13$ & 0.592 \\
\hline $\mathrm{No}^{\dagger}$ & $2(16.7)$ & $68(23.4)$ & 1 & & \\
\hline
\end{tabular}

study population. Out of the 12 patients who developed VTE: 5 patients died, 4 patients died from massive PE, and the remaining patient developed DVT without PE and died of sepsis.

Univariate logistic regression analysis for risk factors with odds ratio (OR) and 95\% confidence interval (CI) for risk factors revealed 6 significant risk factors (Table 2): obesity, need for intubation, septicemia, the presence of 4th degree burn, electrical burn, and the burn percentage being over $25 \%$ of the TBSA.

However, after conducting multivariate logistic regression analysis, the electrical type of burn was found to be the only independent significant risk factor for developing VTE (OR 7.62, 95\% CI 1.34-43.28, $p=0.022)$ (Table 3).

Discussion. At our Burn Unit, most burn patients (89\%) received prophylactic anticoagulation. The most important finding from our study is that $3.9 \%$ of patients developed VTE; and all those who developed VTE were on anticoagulants. Furthermore, the multivariate logistic regression analysis showed that electric burns were the only independent significant risk factor of developing VTE. However, the total number of patients who developed VTE was only 12 patients; and the univariate logistic regression analysis for risk factors identified another 5 risk factors: large (defined as over $25 \%$ of the body surface area) burns, the need for intubation, the occurrence of septicemia, the presence of 4th degree burn, and obesity. The lack of significance of these risk factors using the multivariate logistic regression analysis may be related to the small total number of patients who developed VTE.

Table 3 - Multivariate logistic regression analysis for risk factors of venous thromboembolism among burn patients admitted to the burn unit.

\begin{tabular}{lccc}
\hline Risk factors & OR & $\begin{array}{c}95 \% \text { CI for } \\
\text { OR }\end{array}$ & $P$-value \\
\hline Obesity & 3.25 & $0.72-14.62$ & 0.125 \\
Patient intubated & 0.79 & $0.14-4.60$ & 0.795 \\
Septicemia & 0.18 & $0.03-1.30$ & 0.089 \\
$\begin{array}{l}\text { Degree of burn } \\
\text { 2nd degree }{ }^{\dagger}\end{array}$ & 1.00 & & \\
3rd degree & 0.44 & $0.08-2.57$ & 0.362 \\
4th degree & 7.71 & $0.45-133.30$ & 0.160 \\
Type of burn & & & \\
Flame burn & & & \\
Chemical burn & 3.19 & $0.22-46.08$ & 0.394 \\
Electrical burn & 7.62 & $1.34-43.28$ & $0.022^{*}$ \\
TBSA $\leq 25^{\dagger}$ & 1.00 & \\
TBSA $>25$ & 3.17 & $0.30-33.57$ & 0.338 \\
\hline *Significant $p$ value $(p<0.05) .{ }^{\dagger}$ Used as reference, OR - odds ratio, \\
\multicolumn{4}{l}{ CI- confidence interval } \\
\hline
\end{tabular}


Electrically injured patients have several risk factors for DVT including the presence of fourth degree burn, long ICU stays, multiple operations, and lengthened immobility. ${ }^{11}$ Our study showed that an electrical burn is the most significant risk factor for developing VTE. Obesity is generally regarded as a risk factor for VTE. In literature, obese burn patients were found to have a significant risk factor for VTE in some studies; ${ }^{12}$ but not in others. ${ }^{4,10}$ After conducting a multivariate logistic regression analysis, obesity was not found to be a significant risk factor in our study. Fourth degree burns are usually seen in electrical and chemical burns as well as in severe flame burns (usually entrapped or intoxicated patients). We did not find a study in literature that specifically considered the 4th degree burn as a risk factor. In our study, this was a risk factor using the univariate analysis. Hence, we recommend studying this factor further in a larger study population.

Septicemia by itself represents a risk factor that was not acknowledged in most previous studies. ${ }^{13}$ In septicemia, Neutrophil Extracellular Traps are significantly involved in trapping bacteria in blood and providing an environment for the formation of a thrombus. ${ }^{13}$ Furthermore, burn patients who develop septicemia usually have other risk factors for VTE such as prolonged ICU stay, prolonged immobilization, and the need for intubation. In our study, septicemia was a risk factor using the univariate analysis. We also recommend studying this factor further in a larger study population.

Intubated patients usually have many risk factors that may cause them to have VTE including immobilization, sedation, the use of vasopressors, and having inserted central venous catheters. ${ }^{14}$ Prolonged intubation in burn patients is considered as a risk factor for VTE. ${ }^{15,16}$ In our study intubation was found to be a significant factor with the univariate but not in the multivariate analysis.

Major burns (over 25\% body surface area) are usually associated with several VTE risk factors such as intubation, septicemia, repeated blood transfusions, repeated surgery, and lengthened immobilization. ${ }^{9,16}$ However, other studies in literature as well as our study did not find the percentage of burn to be an independent risk factor for VTE. ${ }^{4,15}$

Finally, blood transfusion was not found to be a risk factor for VTE in our study. However, other studies showed that transfusing more than 4 units of blood was a significant risk factor. ${ }^{4}$
Study limitations. Being retrospective and the relatively small number of patients are the limitation of the study. In addition, studying the patients of a single center limits generalization of the results.

In conclusion, the study investigated the rate and risk factors of VTE in burn patients over 7 years. It is peculiar to other studies in highlighting the presence of electrical burns as the most important risk for VTE in burn patients while taking into consideration the wide confidence interval it has due to the small number of VTE cases. The Caprini score has been validated as a predictor of VTE risk in medical and surgical patients. The score is currently in use in most hospitals. We believe that the presence of electrical burn should be added to the score when calculating the score for burn patients.

Acknowledgment. We would like to thank the King Abdullah International Medical Research Center, Riyadh, Saudi Arabia for the English language editing.

\section{References}

1. Sebastian R, Ghanem O, DiRoma F, Milner S, Price L. Pulmonary embolism in burns, is there an evidence based prophylactic recommendation? Case report and review of literature. Burns 2015; 41: e4-e7.

2. Rue LW 3rd, Cioff WG Jr, Rush R, McManus WF, Pruitt BA Jr. Thromboembolic complications in thermally injured patients. World J Surg 1992; 16: 1151-1154.

3. Abedi N, Papp A. A survey of current practice patterns in prophylaxis against venous thromboembolism (VTE) and gastrointestinal (GI) ulceration among Canadian burn centers. Burns 2011; 37: 1182-1186.

4. Wibbenmeyer LA, Hoballah JJ, Amelon MJ, Chang PX, Loret de Mola RM, Lewis RD, et al. The prevalence of venous thromboembolism of the lower extremity among thermally injured patients determined by duplex sonography. J Trauma 2003; 55: 1162-1167.

5. Fecher AM, O’Mara MS, Goldfarb IW, Slater H, Garvin R, Birdas TJ, et al. Analysis of deep vein thrombosis in burn patients. Burns 2004; 30: 591-593.

6. Mullins F, Mian M, Jenkins D, Brandigi C, Shaver J, Friedman $\mathrm{B}$, et al. Thromboembolic complications in burn patients and associated risk factors. J Burn Care Res 2013; 34: 355-360.

7. Barret J, Dziewulski P. Complications of the hypercoagulable status in burn injury. Burns 2006; 32: 1005-1008.

8. Pachter H, Riles T. Low dose heparin: bleeding and wound complications in the surgical patient. A prospective randomized study. Ann Surg 1977; 186: 669-674.

9. Satahoo S, Parikh P, Naranjo D, Davis J, Duncan R, Pizano L, et al. Are burn patients really at risk for thrombotic events? J Burn Care Res 2015; 36: 100-104.

10. Wahl W, Brandt M, Ahrns K, Zajkowski P, Proctor M, Wakefield $\mathrm{T}$, et al. Venous thrombosis incidence in burn patients: Preliminary results of a prospective study. J Burn Care 2001; 23: 97-92. 
11. Pannucci C, Diaz J, Wahl W. Temporal changes in deep venous thrombosis risk after electrical injury. J Burn Care Res 2011; 32: 442-446.

12. Harrington DT, Mozingo DW, Cancio L, Bird P, Jordan B, Goodwin CW. Thermally injured patients are at significant risk for thromboembolic complications. J Trauma 2001; 50: 495-499.

13. Piazza G. Beyond Virchow's Triad: Does cardiovascular inflammation explain the recurrent nature of venous thromboembolism? Vascular Medicin. 2015; 20: 102-104.
14. Minet C, Potton L, Bonadona A, Hamidfar-Roy R, Somohano CA, Lugosi M, et al. Venous thromboembolism in the ICU: main characteristics, diagnosis and thromboprophylaxis. Crit Care 2015; 19: 287.

15. Pannucci C, Osborne N, Park H, Wahl W. Acquired inpatient risk factors for venous thromboembolism after thermal injury. $J$ Burn Care Res 2012; 33: 84-88.

16. Pannucci C, Osborne N, Wahl W. Venous thromboembolism in thermally injured patients: analysis of the National Burn Repository. J Burn Care Res 2011; 32: 6-12.

\section{www.smj.org.sa}

Saudi Medical Journal Online features

* Instructions to Authors

* Uniform Requirements

* STARD

* Free access to the Journal's Current issue

* Future Contents

* Advertising and Subscription Information

All Subscribers have access to full text articles in HTML and PDF format. Abstracts and Editorials are available to all Online Guests free of charge. 